\title{
Celery extract inhibits mouse CYP2A5 and human CYP2A6 activities via different mechanisms
}

\author{
XIAO DENG, QIANGHONG PU, ERHAO WANG and CHAO YU \\ Institute of Life Sciences, Chongqing Medical University, Chongqing 400016, P.R. China
}

Received July 29, 2015; Accepted September 13, 2016

DOI: $10.3892 / \mathrm{ol} .2016 .5317$

\begin{abstract}
Human cytochrome P450 (CYP) 2A6 participates in the metabolism of nicotine and precarcinogens, thus the deliberate inhibition of CYP2A6 may reduce cigarette consumption and therefore reduce the risk of developing the types of cancer associated with smoking. The inhibitory effects and mechanisms of celery (Apium graveolens) extract on mouse CYP2A5 and human CYP2A6 activity remain unclear. These effects were investigated in mouse and human liver microsomes using coumarin 7-hydroxylation in a probe reaction. Celery extract reduced CYP2A5 and CYP2A6 activities in vitro in a dose-dependent manner. In vivo experiments also showed that celery extract markedly decreased CYP2A5 activity. The inhibition of celery extract on CYP2A5 was time- and nicotinamide adenine dinucleotide phosphate (NADPH)-independent, and was markedly reduced by ultracentrifugation. Additionally, the inhibition of celery extract on CYP2A6 was time and NADPH-dependent. Levels of inhibition were characterized by a $\mathrm{K}_{\mathrm{i}}$, the measure of the tightness of bonds between the enzyme and its inhibitor, of $266.4 \mu \mathrm{g} / \mathrm{ml}$ for CYP2A5, and a $\mathrm{K}_{\mathrm{i}}$ of $1,018 \mu \mathrm{g} / \mathrm{ml}$ and $\mathrm{K}_{\text {inact }}$ of $0.3 / \mathrm{min}$ for CYP2A6. $\mathrm{K}_{\text {inact }}$ is the maximal rate of enzyme inactivation at a saturating concentration of inhibitor. The coumarin derivative 5-methoxypsoralen present in celery extract did not solely to the inhibition of CYP2A5/6 activity. In conclusion, celery extract inhibited the levels of mouse CYP2A5 and human CYP2A6 activity via different mechanisms: Mixed competitive inhibition for CYP2A5 and mechanism-based inhibition for CYP2A6.
\end{abstract}

\section{Introduction}

Human cytochrome P450 (CYP) 2A6 and mouse CYP2A5 share $82 \%$ of their amino acid sequences, and belong to the cytochrome $2 \mathrm{~A}$ family of enzymes $(1,2)$. The two enzymes

Correspondence to: Professor Chao Yu, Institute of Life Sciences, Chongqing Medical University, 1 Yixueyuan Road, Yuzhong, Chongqing 400016, P.R. China

E-mail: yuchaom@163.com

Key words: Apium graveolens, coumarin 7-hydroxylase, mechanism-based inhibition are mainly expressed in the liver and are involved in the metabolization of a series of xenobiotics including nicotine and methoxyflurane, and in activating a variety of precarcinogens such as afltoxin B1, tobacco-specific nitrosamines, $\mathrm{N}$-nitrosodiethylamine, 4-(methylnitrosamino)-1(3-pyridyl)-1butanone and N-nitrosonornicotine (3-5). Human CYP2A6 has received more attention, as it is a key enzyme with respect to the biotransformation of nicotine into inactive cotinine and the activation of tobacco-specific nitrosamines (5). Thus, the deliberate inhibition of CYP2A6 activity is a potential strategy in chemoprevention, since CYP2A6 inhibition has been found to prolong the biological lifetime of nicotine, therefore decreasing cigarette consumption (6). CYP2A6 has also been found to reduce the number of active carcinogens transferred from tobacco (7).

Celery (Apium graveolens) belongs to the umbelliferae family and is widely used in food and for medicinal purposes. Studies have found that celery extracts can exert beneficial effects, including antioxidant, hypoglycemic, hypolipidemic and anti-platelet aggregation effects $(8,9)$. A previous study demonstrated that the juice extracted from Apium graveolen and Petroselinum sativum extended the analgesic effect of aminopyrine and paracetamol, suggesting that the juice inhibited CYP activity in the liver, including that of aminopyrine N-demethylase (10). Whether or not other CYP enzymes are inhibited remains unclear. Coumarin 7-hydroxylation is catalyzed by CYP2A enzymes and is thus considered to be a specific indicator for the presence of CYP2A enzymes, such as CYP2A5 in mice and CYP2A6 and CYP2A13 in humans (11). Notably, celery extract contains a number of coumarin derivatives including furocoumarins and pyranocoumarins. The present study therefore postulates that celery extract may inhibit CYP2A5/6-mediated coumarin 7-hydrolase activity according to a structure-activity association. To the best of our knowledge, there have been few studies on the inhibitory effects and mechanisms of celery extract on CYP2A5/6. Therefore, the objectives of the present study are to assess the inhibition potency of celery extract on CYP2A5/6 activity using mouse and human liver microsomes, and to analyze the inhibition mechanisms of celery extract using time- and nicotinamide adenine dinucleotide phosphate (NADPH)dependent, ultracentrifugation tests. Finally, the present study will clarify whether or not furocoumarin 5-methoxypsoralen (bergapten), present in celery extract, is the predominant inhibitor of CYP2A5/6. 


\section{Materials and methods}

Chemicals and reagents. Coumarin, 4-methylumbelliferyl and NADPH were purchased from Sigma-Aldrich (EMD Millipore, Billerica, MA, USA). The 7-hydroxycoumarin was purchased from Aladdin Shanghai Biochemical Tech Co. Ltd. (Shanghai, China). Human liver microsomes were purchased from the Research Institute for Liver Diseases Co. Ltd. (Shanghai, China). Ultra-pure water was prepared with Milli-Q (EMD Millipore). High performance liquid chromatography (HPLC)-grade acetonitrile was obtained from Tedia (Fairfield, OH, USA). All other reagents were of analytical grade or above and commercially available.

Celery extract preparation. In total, $15 \mathrm{~g}$ fresh celery was used to make celery powder by pulverizeration. Celery powder $(4 \mathrm{~g})$ was extracted with $60 \mathrm{ml}$ petroleum ether at $40^{\circ} \mathrm{C}$ following saturation for three times. The extracts were filtered and evaporated resulting in a volume of $\sim 5 \mathrm{ml}$, and finally metered to $10 \mathrm{ml}$ with petroleum ether. The final liquid extract was stored at $4^{\circ} \mathrm{C}$ prior to use.

Animal experiments. All animal care and experimental protocols were approved by the Animal Center of Chongqing Medical University (Chongqing, China). Male Swiss mice (15-25 g, age 7 weeks) were purchased from the Animal Center of Chongqing Medical University. The mice were housed in a temperature-controlled room, with free access to rodent chow and water and a 12:12 h light-dark cycle. Subsequent to a one-week acclimation period, celery liquid extract $(0.2 \mathrm{mg} / \mathrm{l}$; $\mathrm{n}=5$ ) or solvent $(\mathrm{n}=5)$ was orally administered twice to the mice, as previously described by Jakovljevic et al (10). The mice were sacrificed $2 \mathrm{~h}$ after the last administration, and their livers were harvested. The liver microsomes were then prepared and the CYP2A5 activity was determined as described below.

Mouse liver microsomes preparation. Mouse liver microsomes were prepared from male Swiss mice (15-25 g, age 6-8 weeks) as described by Pinto et al (12). The protein concentrations of microsomal samples were determined by the Lowry method (13).

Assay of coumarin 7-hydroxylation in liver microsomes. A coumarin 7-hydroxylation assay was performed as described by Aitio (14) with certain modifications. The procedure was as follows. The incubation mixture contained mouse $(0.4 \mathrm{mg} / \mathrm{l})$ or human liver microsomes $(0.1 \mathrm{mg} / \mathrm{l}$; a kind gift from Dr Guo of Chongqing Medical University), coumarin $(100 \mathrm{mmol} / \mathrm{l})$ and $100 \mathrm{mM}$ potassium phosphate buffer ( $\mathrm{pH}$ 7.4) creating a total volume of $180 \mu \mathrm{l}$. Subsequent to $3 \mathrm{~min}$ pre-incubation, the reaction was initiated by the addition of $20 \mu 1$ of $10 \mathrm{mM}$ NADPH and performed for either $20 \mathrm{~min}$ (mouse liver microsomes) or $15 \mathrm{~min}$ (human liver microsomes) at $37^{\circ} \mathrm{C}$, and terminated by the addition of $200 \mu \mathrm{l}$ of $100 \mathrm{nM}$ 4-methylumbelliferyl, an internal standard, in ice-cold acetonitrile. The resulting samples were then centrifuged at $10,000 \times g$ for $15 \mathrm{~min}$. The product formed, 7-hydroxycoumarin (umbelliferone), was quantified by HPLC, as previously described by Farooq et al (15). Protein concentration and incubation time were optimized and product formation was linear under the aforementioned conditions.
Microsomal CYP2A5/6 inactivation assay. To examine the concentration-dependence and the value of the concentration of the inhibitor where binding is reduced by half $\left(\mathrm{IC}_{50}\right)$ of CYP2A 5/6 inactivation, celery extracts of increasing concentrations were pre-incubated with the incubation mixture at $37^{\circ} \mathrm{C}$ for $3 \mathrm{~min}$, and the same pre-incubation mixture was added to the NADPH solution to start the reaction. The reaction was performed as aforementioned to determine the level of residual coumarin 7-hydroxylase activity.

To test the time-dependence of CYP2A5 inactivation, celery extracts of increasing concentrations $(0,200,400$, 600,800 and $1,000 \mu \mathrm{g} / \mathrm{ml}$ ) were pre-incubated with the liver microsomes at $37^{\circ} \mathrm{C}$ for the selected time points. At selected time points (0, 3, 6 and $9 \mathrm{~min}), 20 \mu \mathrm{l}$ of mixture was added to the NADPH solution to start the reaction. The reaction was performed as aforementioned to determine the residual coumarin 7-hydroxylase activity.

To investigate the dependence of CYP2A5/6 inactivation on NADPH, celery extract was pre-incubated with the mouse or human liver microsomes and $100 \mathrm{mM}$ of potassium phosphate buffer ( $\mathrm{pH} \mathrm{7.4)}$ at $37^{\circ} \mathrm{C}$ for $30 \mathrm{~min}$ in the presence or absence of NADPH. The reaction was then initiated via the addition of coumarin and NADPH and performed as aforementioned to determine the level of residual coumarin 7-hydroxylase activity.

$I C_{50}$ shift assay. The $\mathrm{IC}_{50}$ shift assay was performed as described by Perloff et al (16) with certain modifications. Celery extract of increasing concentration was pre-incubated with the human liver microsomes, NADPH and potassium phosphate buffer for 0 or $30 \mathrm{~min}$. Subsequent to pre-incubation, the pre-incubation mixtures were then transferred to the secondary incubations containing coumarin and NADPH to initiate the reaction. This transferal took place at approximately the $\mathrm{K}_{\mathrm{m}}$, which is the concentration of substrate required to produce $50 \%$ of the $\mathrm{V}_{\max }$ (maximum velocity or rate at which the enzyme catalyzed the reaction). The reaction was performed as aforementioned to determine the level of residual coumarin 7-hydroxylase activity and the value of $\mathrm{IC}_{50}$.

Ultracentrifugation test. The ultracentrifugation test was performed as described by Lee et al (17) with certain modifications. To evaluate the reversibility of the drug-enzyme interaction, the celery extract was incubated with the mouse liver microsomes and a potassium phosphate buffer ( $\mathrm{pH}$ 7.4) at $37^{\circ} \mathrm{C}$ for $30 \mathrm{~min}$. The microsomes were re-isolated by ultracentrifugation of the pre-incubation mixtures at $80,000 \times \mathrm{x} g$ for $60 \mathrm{~min}$ at $4^{\circ} \mathrm{C}$. The microsomes were washed twice with $0.1 \mathrm{M}$ potassium phosphate buffer $(\mathrm{pH}$ 7.4). The residual CYP2A5 activity was then determined as aforementioned.

Data analysis. Data is expressed as the mean \pm standard error of the mean. All tests were performed at least 3 times. The enzyme kinetic and the Lineweaver-Burk plot analyses were performed with GraphPad Prism 5.0 (GraphPad software, Inc., La Jolla, CA, USA). Statistical significance was calculated using a one-tailed Student t-test, or a one-way analysis of variance and Dunnet's test as a post-hoc test. $\mathrm{P}<0.05$ was considered to indicate a statistically significant difference. 

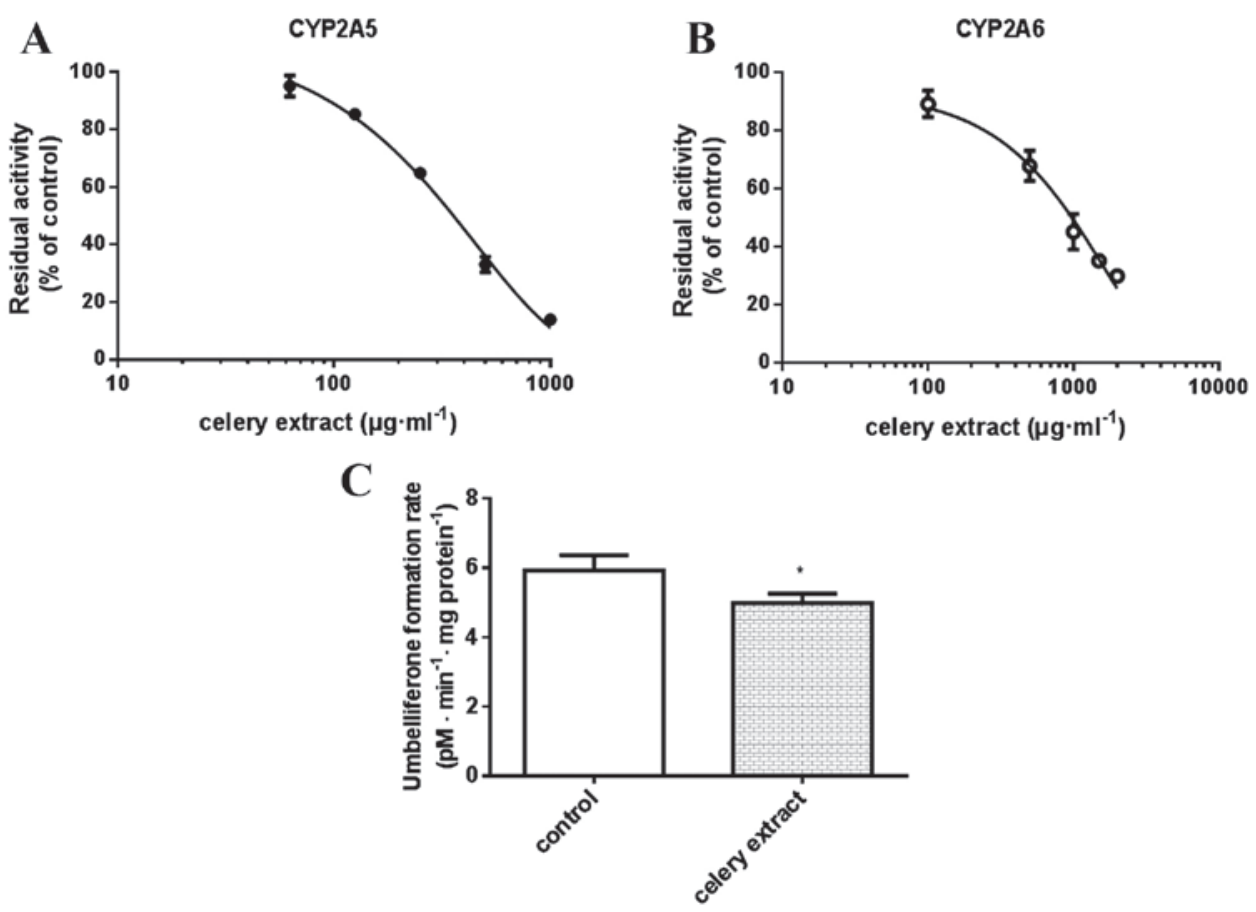

Figure 1. Inhibitory effects of celery extract on mouse CYP2A5 and human CYP2A6 activities. (A) Celery extract concentration-dependently inactivated CYP2A5 and (B) CYP2A6 in the mouse and human liver microsomes, respectively. (C) Celery extract significantly decreased the mouse CYP2A5 activity in vivo. Data is expressed as the mean \pm standard deviation $(\mathrm{n}=3)$. ${ }^{*} \mathrm{P}<0.05$. CYP, cytochrome $\mathrm{P} 450$.

A

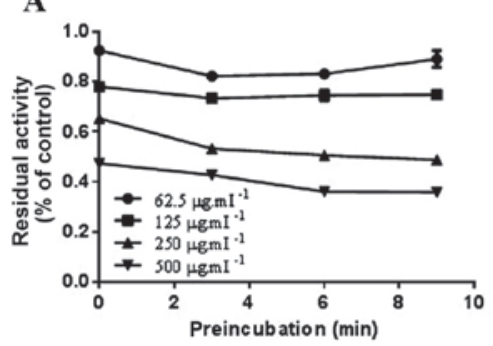

B

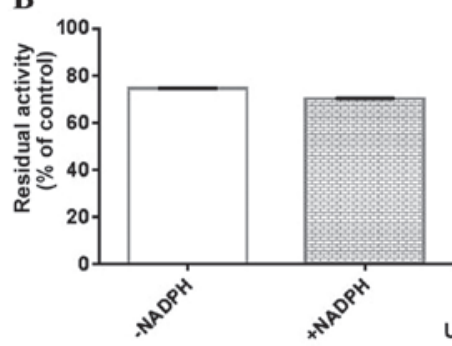

C

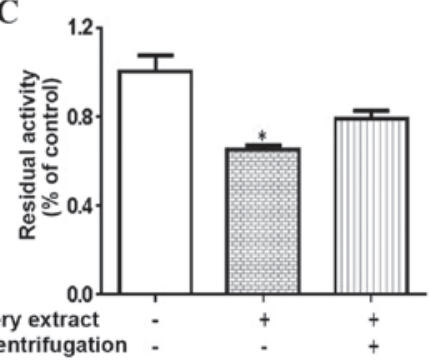

D
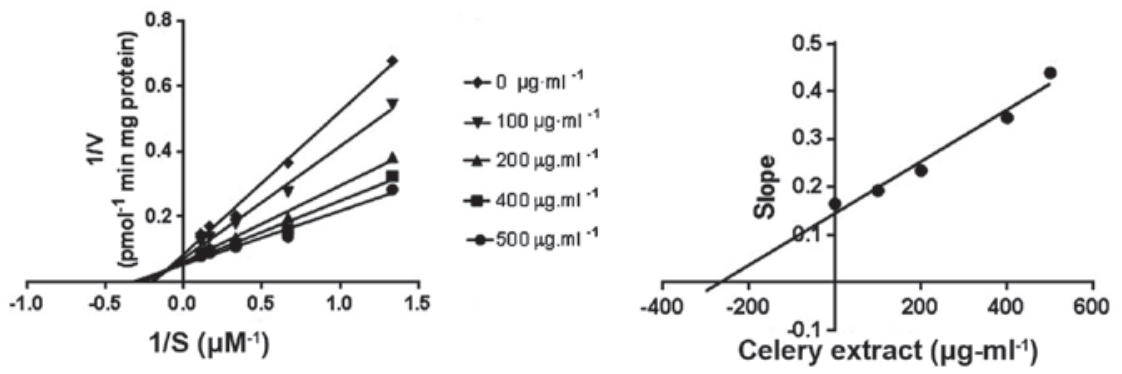

Figure 2. Reversible inhibition of CYP2A5 by celery extract in mouse liver microsomes. (A) Time-independent inhibition of celery extract on CYP2A5. (B) NADPH-independent inhibition of celery extract on CYP2A5. (C) CYP2A5 activity was recovered by ultracentrifugation. (D) Lineweaver-Burk and its second plot for the inhibition of CYP2A5 activity by different concentrations of celery extract in the mouse liver microsomes. The inhibition of celery extract on CYP2A5 activity is best described as a competitive/noncompetitive mechanism. Data is expressed as the mean \pm standard deviation ( $\mathrm{n}=3$ ). ${ }^{*} \mathrm{P}<0.05$. CYP, cytochrome P450; NADPH, nicotinamide adenine dinucleotide phosphate.

\section{Results}

Celery extract inhibits CYP2A5/6 activity. The potential inhibitory effect of celery extract on CYP2A $5 / 6$ activity was investigated in the mouse and human liver microsomes, using coumarin as a specific probe. The $\mathrm{K}_{\mathrm{m}}$ and $\mathrm{V}_{\max }$ values for the coumarin 7-hydroxylation of CYP2A5 were $1.4 \mu \mathrm{M}$ and $15.3 \mathrm{pM}$ umbelliferone $/ \mathrm{min} / \mathrm{mg}$ microsomal protein. The $\mathrm{K}_{\mathrm{m}}$ and $\mathrm{V}_{\max }$ values for CYP2A6 were $1.9 \mu \mathrm{M}$ and $48.3 \mathrm{pM}$ umbelliferone $/ \mathrm{min} / \mathrm{mg}$ microsomal protein. When celery extract was added to the incubation mixture at varying concentrations, coumarin 7-hydroxylation was concentration-dependently 
A

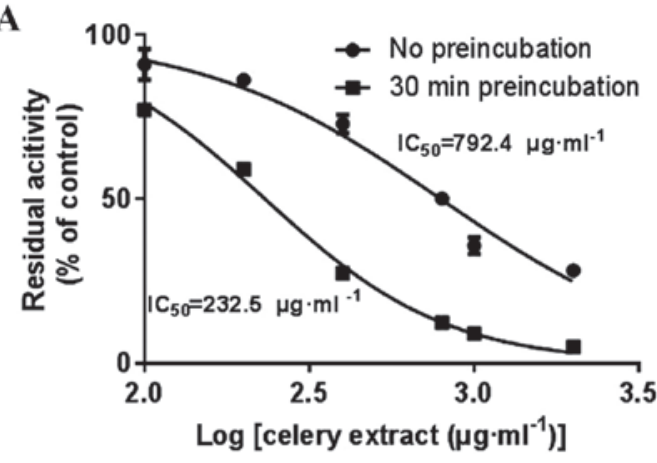

C

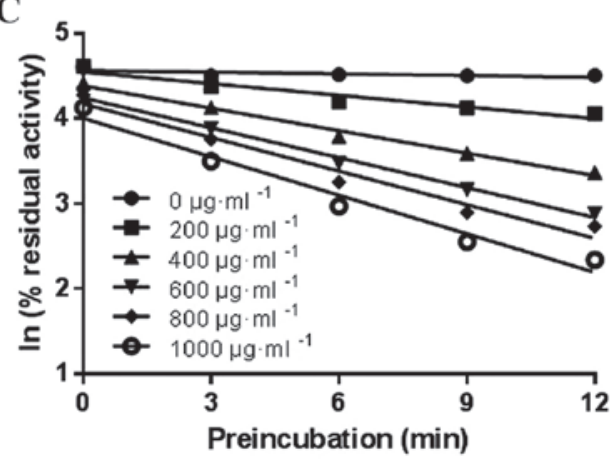

B
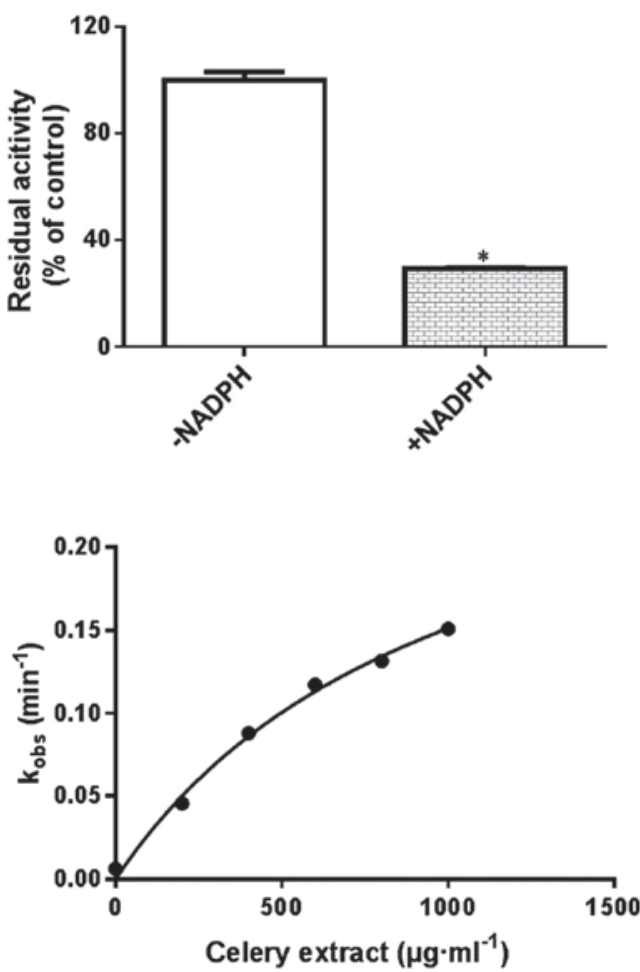

Figure 3. Irreversible inhibition of CYP2A6 by celery extract in human liver microsomes. (A) A 3.4-fold IC ${ }_{50}$ shift showed time-dependent inactivation of CYP2A6 by celery extract. (B) NADPH-dependent inhibition of celery extract on CYP2A6. (C) Pre-incubation time- and concentration-dependent inhibition of CYP2A6 by celery extract in the human liver microsomes. The inactivation rate $\left(\mathrm{k}_{\mathrm{obs}}\right)$ initially observed was determined by linear regression analysis of the natural logarithm of the percentage remaining activity vs. pre-incubation time. The $\mathrm{K}_{\mathrm{i}}$ and $\mathrm{K}_{\text {inact }}$ values were determined using nonlinear regression analysis of the $k_{\mathrm{obs}}$ value vs. celery extract concentration. Data is expressed as the mean \pm standard deviation ( $\mathrm{n}=3$ ). ${ }^{*} \mathrm{P}<0.05$. CYP, cytochrome $\mathrm{P} 450$; $\mathrm{NADPH}$, nicotinamide adenine dinucleotide phosphate; $\mathrm{IC}_{50}$, value of the concentration of the inhibitor where binding is reduced by half; $\mathrm{K}_{\mathrm{i}}$, measure of the tightness of bonds between an enzyme and its inhibitor; $\mathrm{K}_{\text {inact }}$, maximal rate of enzyme inactivation at a saturating concentration of inhibitor.
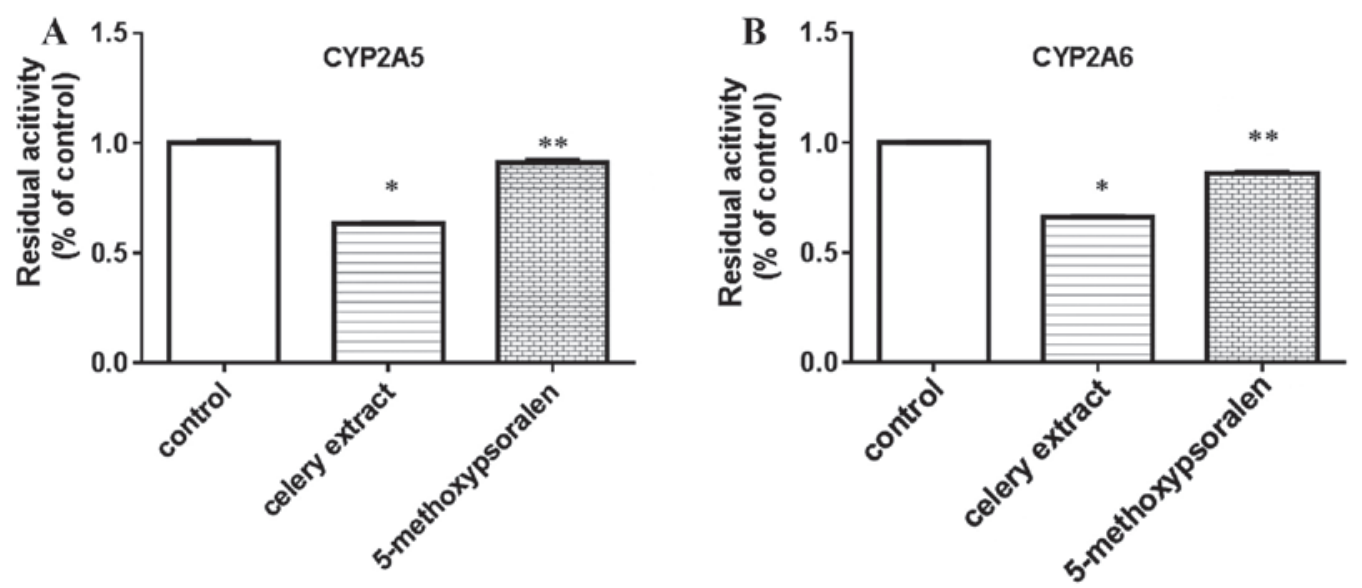

Figure 4. 5-methoxypsoralen reduced (A) CYP2A5 and (B) CYP2A6 activity by less than celery extract in the mouse and human liver microsomes, respectively. Data is expressed as the mean \pm standard deviation $(\mathrm{n}=3)$. ${ }^{*} \mathrm{P}<0.05$ vs. control; ${ }^{* *} \mathrm{P}<0.05$ vs. celery extract. CYP, cytochrome $\mathrm{P} 450$.

inhibited with $\mathrm{IC}_{50}$ values of 345.1 and $888.7 \mu \mathrm{g} / \mathrm{ml}$, respectively, for CYP2A5 and CYP2A6 (Fig. 1A and B). The animal experiment verified that celery extract significantly decreased the CYP2A5 activity by $16 \%(\mathrm{P}=0.039)$ (Fig. 1C). Therefore, celery extract was found to be an inhibitor of CYP2A5/6.

Celery extract reversibly inhibits mouse CYP2A5 but irreversibly inhibits human CYP2A6. The present study used kinetic inhibition studies to investigate the inhibition modes of the celery extract on CYP2A5/6. Time-dependent inhibition of CYP2A5 with celery extract was not observed (Fig. 2A). Celery extract samples that were pre-incubated in the presence and absence of NADPH demonstrated the same levels of CYP2A5 activity (Fig. 2B), suggesting that CYP2A5 inactivation occurs independently of NADPH. The ultracentrifugation test demonstrated that CYP2A5 activity returned to a normal level if the incubation mixture was ultracentrifuged at $80,000 \times \mathrm{g}$ for $60 \mathrm{~min}$, indicating reversible non-covalent binding of the 
inhibitor to CYP2A5, and the reversible inhibition of celery extract to CYP2A5 (Fig. 2C). The aforementioned results suggest that celery extract is a reversible inhibitor for CYP2A5. The Lineweaver-Burk plot demonstrates that the lines intersect on the second quadrant, indicating that the inhibition of CYP2A5 was mixed competitive/noncompetitive, with a $\mathrm{K}_{\mathrm{i}}$ value of $266.4 \mu \mathrm{g} / \mathrm{ml}$ (Fig. 2D).

By contrast, time-dependent inhibition of CYP2A6 by celery extract was observed, since a 3.4-fold shift for CYP2A6 was observed in the $\mathrm{IC}_{50}$-shift experiments (Fig. 3A). Samples that were pre-incubated with celery extract in the presence of NADPH exhibited less activity compared with those incubated in the absence of NADPH $(\mathrm{P}=0.019$; Fig. $3 \mathrm{~B})$, suggesting the dependence of CYP2A6 inactivation on NADPH. Therefore, the aforementioned results revealed that the inactivation of CYP2A6 activity by celery extract is dependent on celery concentration, time and NADPH, indicating that celery extract is an irreversible inhibitor for CYP2A6. To characterize the irreversible inhibition of CYP2A6 by celery extract, the $\mathrm{K}_{\mathrm{i}}$ and $\mathrm{K}_{\text {inact }}$ values were determined. The $\mathrm{K}_{\mathrm{i}}$ and $\mathrm{K}_{\text {inact }}$ values were $1,018 \mu \mathrm{g} / \mathrm{ml}$ and 0.3/min, respectively (Fig. 3C).

5-methoxypsoralen reduces less CYP2A5/6 activity compared with celery extract. To explore whether 5-methoxypsoralen, the main component present in celery extract, played a critical role in the inhibition of CYP2A5/6 activity, the present study analyzed the inhibitory effects of 5-methoxypsoralen. Fig. 4 demonstrates that 5-methoxypsoralen $(0.3 \mu \mathrm{M})$ significantly decreased CYP2A5/6 activity $(\mathrm{P}=0.037)$, but to a lesser extent compared with celery extract $(400 \mu \mathrm{g} / \mathrm{ml})$ did, compared with the control $(\mathrm{P}=0.031)$. The results suggest that other coumarin derivatives, besides 5-methoxypsoralen, also contribute to the inhibition of CYP2A5/6 activity.

\section{Discussion}

The present study revealed that celery extract inhibited mouse CYP2A5- and human CYP2A6-mediated coumarin 7-hydroxylase activity via different mechanisms. To explore whether celery extract played a concentration-dependent role on the level of CYP2A5/6 activity, the present study used 3 different concentrations of celery extract. The results demonstrate that celery extract is an inhibitor of CYP2A5/6 (Fig. 1A). For CYP2A5, celery extract behaves as a reversible (mixed competitive and noncompetitive) inhibitor, but for CYP2A6, celery extract behaves as an irreversible inhibitor. In addition, it appears that celery extract is a more potent inhibitor of CYP2A5 compared with CYP2A6, since the $\mathrm{IC}_{50}$ value for CYP2A5 is $\sim 2$-times smaller than that of CYP2A6. A previous study also identified that 8-methoxypsoralen, a furocoumarin present in celery extract, is a more potent inactivator for CYP2A5 compared with for CYP2A6 in vitro, with $\mathrm{IC}_{50}$ values 1.0 and $5.4 \mu \mathrm{M}$, respectively (18).

These differences may be associated with the size of mouse CYP2A5 and human CYP2A6 active sites. The majority of compounds studied, including furocoumarins, metyrapone, miconazole and lactone derivatives, have been found to be stronger suppressors of CYP2A5 activity than of CYP2A6 activity (18-20). Previous studies demonstrated that larger compounds were more powerful inhibitors of CYP2A5 activity than of CYP2A6 activity, suggesting that CYP2A5 has a larger active site compared with CYP2A6 (19,21). Therefore, the compounds in celery extract are more likely to get into, and interact with, the active site of CYP2A5. These differences may be due to amino acid changes in the active sites of these enzymes. A single mutation, whereby phenylalanine 209 is substituted by leucine in the substrate-binding site, changes the specificity of CYP2A5 from coumarin to steroid hydroxylation $(19,22)$. Therefore, similar amino acid sequences are shared in CYP2A5/6 enzymes, but small amino acid changes between active sites affect their substrate-recognition ability. The present study supports previous studies that revealed that CYP2A5 and CYP2A6 have different substrates and inhibitor specificities $(21,23)$.

In addition, the present study demonstrated that the coumarin derivative 5-methoxypsoralen in celery extract markedly inhibited CYP2A5/6 activity, but to a lesser extent than celery extract did, suggesting that other coumarin derivatives besides 5-methoxypsoralen also contribute to the inactivation of CYP2A5/6 activity. A coumarin derivative that may be present in celery extract was considered to be the furocoumarin 8-methoxypsoralen, since it is the only well-characterized inhibitor for CYP2A enzymes including CYP2A5 and CYP2A6 and as it also demonstrated distinct inhibition modes for CYP2A5 and CYP2A6 (24-26).

Pilot studies suggested that inactive variations of the CYP2A6 gene have been revealed to markedly increase the bioavailability of nicotine and thus decrease cigarette consumption, and reduce the risk of developing lung cancer due to smoking (27-29). Therefore, CYP2A6 inhibitors may act as promising adjunct drugs in smoking cessation therapy. Whether celery extract, as a CYP2A6 inhibitor, could affect nicotine kinetics in vivo and therefore that affects smoking behaviors must be investigated in another study.

In conclusion, the present study suggests that celery extract inhibits mouse CYP2A5 and human CYP2A6 via different mechanisms, suggesting that the mouse model is not applicable for future studies involving the inhibition of CYP2A enzymes by celery extract. Future studies must be performed to identify which components of celery extract play the main role in the inhibition of CYP2A enzyme activity, and to investigate whether the deliberate inhibition of CYP2A6 by celery extract can modulate smoking behavior and therefore reduce the risk of lung cancer.

\section{Acknowledgements}

This study was supported by the National Nature Science Foundation of China (grant no. 81370403) and Specialized Research Fund for the Doctoral Program of Higher Education (grant no. 20125503110008).

\section{References}

1. Raunio H and Rahnasto-Rilla M: CYP2A6: Genetics, structure, regulation, and function. Drug Metabol Drug Interact 27: 73-88, 2012.

2. Raunio H, Rautio A and Pelkonen O: The CYP2A subfamily: Function, expression and genetic polymorphism. IARC Sci Publ 148: 197-207, 1999.

3. Pelkonen P, Lang MA, Wild CP, Negishi M and Juvonen RO: Activation of aflatoxin B1 by mouse CYP2A enzymes and cytotoxicity in recombinant yeast cells. Eur J Pharmacol 292: 67-73, 1994. 
4. Camus AM, Geneste O, Honkakoski P, Béréziat JC, Henderson CJ, Wolf CR, Bartsch H and Lang MA: High variability of nitrosamine metabolism among individuals: Role of cytochromes P450 $2 \mathrm{~A} 6$ and 2E1 in the dealkylation of N-nitrosodimethylamine and N-nitrosodiethylamine in mice and humans. Mol Carcinog 7: 268-275, 1993.

5. Oscarson M: Genetic polymorphisms in the cytochrome P450 2A6 (CYP2A6) gene: Implications for interindividual differences in nicotine metabolism: Drug Metab Dispos 29: 91-95, 2001.

6. Tyndale RF and Sellers EM: Variable CYP2A6-mediated nicotine metabolism alters smoking behavior and risk. Drug Metab Dispos 29: 548-552, 2001.

7. Miyamoto M, Umetsu Y, Dosaka-Akita H, Sawamura Y, Yokota J, Kunitoh H, Nemoto N, Sato K, Ariyoshi N and Kamataki T: CYP2A6 gene deletion reduces susceptibility to lung cancer. Biochem Biophys Res Commun 261: 658-660, 1999.

8. Sowbhagya HB: Chemistry, technology, and nutraceutical functions of celery (Apium graveolens L.): An overview. Crit Rev Food Sci Nutr 54: 389-398, 2014

9. Madkour NK: Beneficial role of celery oil in lowering the di(2-ethylhexyl) phthalate-induced testicular damage. Toxicol Ind Health 30: 861-872, 2014.

10. Jakovljevic V, Raskovic A, Popovic M and Sabo J: The effect of celery and parsley juices on pharmacodynamic activity of drugs involving cytochrome P450 in their metabolism. Eur J Drug Metab Pharmacokinet 27: 153-156, 2002.

11. Lowry OH, Rosebrough NJ, Farr AL and Randall RJ: Protein measurement with the Folin phenol reagent. Biol Chem 193: 265-274, 1951.

12. Pinto LF, Moraes E, Albano RM, Silva MC, Godoy W, Glisovic T and Lang MA: Rat oesophageal cytochrome P450 (CYP) monooxygenase system: Comparison to the liver and relevance in $\mathrm{N}$-nitrosodiethylamine carcinogenesis. Carcinogenesis 22: $1877-1883,2001$.

13. Lowry OH, Rosebrough NJ, Farr AL and Randall RJ: Protein measurement with the Folin phenol reagent. J Biol Chem 193: 265-275, 1951.

14. Aitio A: A simple and sensitive assay of 7-ethoxycoumarin deethylation. Anal Biochem 85: 488-491, 1978.

15. Farooq S, Shakeel-u-Rehman, Dangroo NA, Priya D, Banday JA, Sangwan PL, Qurishi MA, Koul S and Saxena AK: Isolation, cytotoxicity evaluation and HPLC-quantification of the chemical constituents from Prangos pabularia. PLoS One 9: e108713, 2014

16. Perloff ES, Mason AK, Dehal SS, Blanchard AP, Morgan L, Ho T, Dandeneau A, Crocker RM, Chandler CM, Boily N, et al: Validation of cytochrome P450 time-dependent inhibition assays: A two-time point IC50 shift approach facilitates kinact assay design. Xenobiotica 39: 99-112, 2009.
17. Lee JY,LeeSY,Oh SJ,Lee KH,Jung YS and Kim SK: Assessment of drug-drug interactions caused by metabolism-dependent cytochrome P450 inhibition. Chem Biol Interact 198: 49-56, 2012.

18. Mäenpää J, Sigusch H, Raunio H, Syngelmä T, Vuorela $P$, Vuorela $\mathrm{H}$ and Pelkonen O: Differential inhibition of coumarin 7-hydroxylase activity in mouse and human liver microsomes. Biochem Pharmacol 45: 1035-1042, 1993.

19. Juvonen RO, Gynther J, Pasanen M, Alhava E and Poso A: Pronounced differences in inhibition potency of lactone and non-lactone compounds for mouse and human coumarin 7-hydroxylases (CYP2A5 and CYP2A6). Xenobiotica 30: 81-92, 2000.

20. Wood AW: Genetic regulation of coumarin hydroxylase activity in mice. Biochemical characterization of the enzyme from two inbred strains and their F1 hybrid. J Biol Chem 254: 5641-5646, 1979.

21. Rahnasto M, Raunio H, Poso A and Juvonen RO: More potent inhibition of human CYP2A6 than mouse CYP2A5 enzyme activities by derivatives of phenylethylamine and benzaldehyde. Xenobiotica 33: 529-539, 2003.

22. Lindberg RL and Negishi M: Alteration of mouse cytochrome P450coh substrate specificity by mutation of a single amino-acid residue. Nature 339: 632-634, 1989.

23. Negishi M, Iwasaki M, Juvonen RO and Aida K: Alteration of the substrate specificity of mouse $2 \mathrm{~A} \mathrm{P} 450$ s by the identity of residue-209: Steroid-binding site and orientation. J Steroid Biochem Mol Biol 43: 1031-1036, 1992.

24. Visoni S, Meireles N, Monteiro L, Rossini A and Pinto LF: Different modes of inhibition of mouse Cyp2a5 and rat CYP2A3 by the food-derived 8-methoxypsoralen. Food Chem Toxicol 46: 1190-1195, 2008.

25. Draper AJ, Madan A and Parkinson A: Inhibition of coumarin 7-hydroxylase activity in human liver microsomes. Arch Biochem Biophys 341: 47-61, 1997.

26. von Weymarn LB, Zhang QY, Ding X and Hollenberg PF: Effects of 8-methoxypsoralen on cytochrome P450 2A13. Carcinogenesis 26: 621-629, 2005.

27. Liu YL, Xu Y, Li F, Chen H and Guo SL: CYP2A6 deletion polymorphism is associated with decreased susceptibility of lung cancer in Asian smokers: A meta-analysis. Tumour Biol 34: 2651-2657, 2013.

28. Mwenifumbo JC and Tyndale RF: Genetic variability in CYP2A6 and the pharmacokinetics of nicotine. Pharmacogenomics 8: 1385-1402, 2007.

29. Nakajima M: Smoking behavior and related cancers: The role of CYP2A6 polymorphisms. Curr Opin Mol Ther 9: 538-544, 2007. 